\title{
The influence of lithium and/or selenium on plasma amino acids profile in rats
}

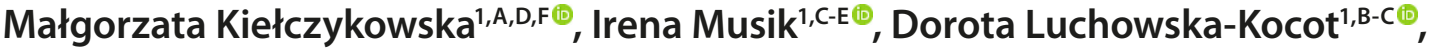 \\ Małgorzata Polz-Dacewicz',E® \\ ${ }^{1}$ Chair and Department of Medical Chemistry, Medical University, Lublin, Poland \\ ${ }^{2}$ Department of Virology with SARS Laboratory, Medical University, Lublin, Poland \\ A - Research concept and design, B - Collection and/or assembly of data, C - Data analysis and interpretation, \\ $D$ - Writing the article, E-Critical revision of the article, F-Final approval of the article
}

Kiełczykowska M, Musik I, Luchowska-Kocot D, Polz-Dacewicz M. The influence of lithium and/or selenium on plasma amino acids profile in rats. Ann Agric Environ Med. 2022; 29(1): 136-142. doi: 10.26444/aaem/139213

\begin{abstract}
Introduction. Selenium belongs to essential microelements and is used in agriculture. Lithium is used in medicine and the possibility of its exposure by environmental pollution has been reported. Both elements have been found to be connected with amino acids metabolism.

Objective. The aim of the study was to compare the effect of lithium and selenium on plasma amino acids in rats, and to evaluate the influence of selenium in organisms exposed to lithium.

Materials and method. The effect of selenium $\left(0.5 \mathrm{mg} / \mathrm{kg}\right.$ b.w., orally as $\left.\mathrm{Na}_{2} \mathrm{SeO}_{3}\right)$ and/or lithium ( $2.7 \mathrm{mg} / \mathrm{kg}$ b.w., orally as $\mathrm{Li}_{2} \mathrm{CO}_{3}$ ) given for 6 weeks on the plasma profile of selected amino acids in rats was studied. The concentrations of amino acids were determined using ion exchange chromatography with the aid of an amino acids analyzer AAA400.

Results. A significant effect of lithium on plasma amino acids profile was found in rats, much greater than for selenium. Selenium treatment slightly increased Tau, Phe, Tyr, Ala, Trp, Ser and Gln, while Lys and Orn were enhanced in a significant way. In contrast, Li-treatment caused a well-marked increase in Phe, Orn, Ala, His, Trp, Asp and Gln, whereas all the others were only slightly increased. Co-treatment resulted in a significant increase in Orn and Trp, a slight enhancement of Phe, Lys and His, while the rest remained unchanged.

Conclusions. A significant effect of lithium alone on plasma amino acids profile in animals was demonstrated, with a much less influence of selenium alone. Co-treatment generally resulted in a slight or no effect. The slight selenium influence seems important regarding its agricultural application and the growing interest in its supplementation. Results concerning lithium could contribute to the research regarding the mechanism of Li action.
\end{abstract}

Key words

rats, lithium, plasma, amino acids, selenium, environmental pollution

\section{Abbreviations}

Met - methionine; Tau - taurine, Phe - phenylalanine; Tyr - tyrosine; Lys - lysine; Orn - ornithine; Gly - glycine; Ala alanine; His - histidine; Trp - tryptophan; Asp - aspartate, Ser - serine; Thr - threonine; Gln - glutamine; Glu - glutamate; GABA - $\gamma$-aminobutyric acid; ALT - alanine amino transferase; AST - aspartate amino transferase

\section{INTRODUCTION}

Selenium belongs to the microelements essential for human beings. According to researchers, a billion people may be affected by its deficit. Since plants are a significant dietary source of selenium the issue of its level in soils represents an important problem as low Se soil levels have been stated in many regions of Asia, Africa and Europe $[1,2]$. Furthermore, despite the fact that it is not regarded as an essential element for plants, many recent studies have indicated its great positive influence on plant growth, including a protective effect against water deficit stress $[1,3,4]$. Observations concerning the influence of selenium on human health, as well as the consequences of its deficit, including deterioration of the immune system, fertility problems, thyroid diseases or cancer risk [5], prompted the Se-enrichment of animal feedstuffs $[6,7]$, as well as the application of selenium compounds in cultivation [8]. Selenium deficit has also been found to be connected with different disorders, including disturbances

Address for correspondence: Małgorzata Kiełczykowska, Chair and Department of Medical Chemistry, Medical University, Chodźki 4 a, 20-093 Lublin, Poland

E-mail: malgorzata.kielczykowska@umlub.pl

Received: 20.04.2021; accepted: 18.06.2021; first published: 09.07.2021 of the nervous system, such as autism [9]. Furthermore, the relationships between metabolic syndrome in schizophrenia and selenium in serum have been observed [10].

However, an excess amount of this element can also show harmful effects, and the safe range of its intake is rather narrow. Researchers have indicated the risk of selenium poisoning resulting from using Se-rich products, by taking an overdose of supplements or environmental pollution caused by industrial and agricultural human activity $[1,2,8,11]$.

However, selenium treatment has been found to exert protective properties against various harmful effects, including the toxicity of chemical substances (e.g. mycotoxins, heavy metals or pesticides) $[7,12,13]$. Selenium-containing proteins have also been reported to influence brain functions through many mechanisms, including affecting GABA, dopamine, acetylcholine and glutamate neurotransmission [14]. Moreover, a selenocompound - ebselen, studied todate as an antioxidant agent and medicine, has been found to possess some lithium-mimetic properties. The results of the performed research has even made the authors suggest that ebselen could be used in mood disorders cure $[15,16$, 17]. The usefulness of such studies has been emphasized, having in view the narrow therapeutic range and numerous 
side-effects of lithium - medicine used in mood disorders therapy [18].

Lithium has been used in medicine since the 1940s when its beneficial action in manic states was found. Since then, many other its pharmacological effects have been discovered, primarily in psychiatry. In addition to its antimanic properties, lithium has been shown to exert anti-depressant and ant-suicidal influence. As a mood stabilizer it is still recommended as the drug of first choice in the treatment of patients with bipolar disorder [19]. Despite large-scale research, the mechanism of the action of lithium remains unclear [20, 21]. However, among other things, research has revealed the relationships between amino acids homeostasis, neurotransmission, and lithium administration $[15,22$, 23]. This is consistent with the fact that, according to some authors, the neurotransmission systems play an important role in the course of affective disorders [24, 25].

Serotonin action has been found to be affected bylithium [26]. The potentialing effect of lithium on anti-depressants actions has also been connected with serotonin neurotransmission [27]. According to other studies, glutamatergic and GABAergic transmission has been suggested to be affected by lithium's action [23]. Additionally, L-glutamic acid monosodium salt has been reported to attenuate $\mathrm{Li}$-induced harmful influence on the development of animal embryos [18]. Research on the influence of lithium on different metabolic processes is all the more warranted because, apart from exposure resulting from medical application, the possibility of exposure to this element due to its presence in drinking water has been reported [28]. Moreover, researchers also indicate the problem of environmental pollution resulting from the use of lithium in batteries [29].

\section{OBJECTIVES}

Taking into account the facts presented above, a study was carried out aimed at evaluating the effect of selenium, lithium, and both these elements administered together, on the plasma concentration of selected amino acids. The aims of the study were: 1) contributing to clarifying the influence of lithium and/or selenium on neurotransmission; 2) comparison of the effect of lithium and selenium on the selected amino acids; 3) evaluating the effect of selenium co-treatment on plasma amino acids profile in organisms exposed to lithium; 4) evaluating the mutual relationships among the changes of the selected amino acids in plasma.

\section{MATERIALS AND METHOD}

The current study was carried on male Wistar rats (130-160 g b.w.), divided randomly into four groups with six animals in each group. The rats were subjected to the proper treatments for six weeks. For the entire experimental period the animals had free access to standard feed LSM (AGROPOL S.J., Motycz, Poland) with no additional lithium and selenium, and ad libitum access to drinking water. Details of the experimental design are shown in Figure 1.

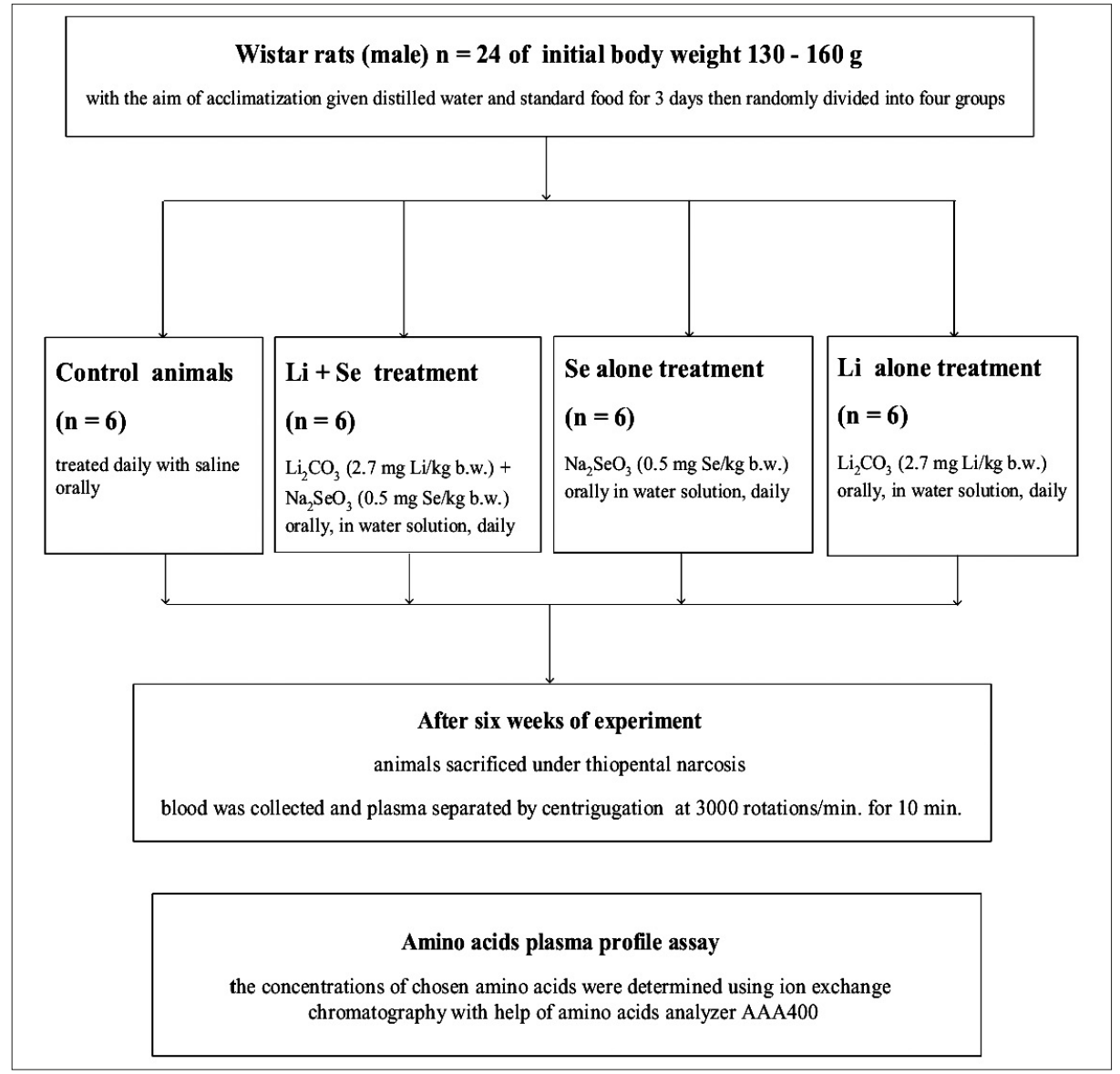

Figure 1. Experimental design 
After the end of the treatment period, the rats were sacrificed, the blood collected, and plasma samples separated. Concentrations of the following amino acids: methionine, taurine, phenylalanine, tyrosine, lysine, ornithine, glycine, alanine, histidine, tryptophan, aspartate, serine, threonine, glutamine, glutamate, and $\gamma$-aminobutyric acid, were determined using ion exchange chromatography with the aid of an amino acids analyzer AAA400.

The study was performed according to statutory bioethical standards and approved by the I Local Ethical Commission of Medical University in Lublin (Acceptance No. 1/2013).

Statistical analysis was performed by the STATISTICA 12.5 PL programme. Shapiro-Wilk test was used for verification of the normality of data distribution. The differences among the experimental groups were determined with application of a one-way analysis of variance (ANOVA), followed by the post-hoc Tukey test for normally distributed variables, or the Kruskal-Wallis one way analysis of variance for non-normally distributed variables. Values were considered significant at $p<0.05$. To evaluate the correlations between the selected amino acids, Spearman correlations coefficients, applied in cases of non-normal distribution, were determined.

\section{RESULTS}

Lithium insignificantly increased Met in comparison with control $(\mathrm{p}=0.2240)$. Selenium, both given alone and in co-treatment with lithium, did not change the plasma concentration ( $p=1.0000$ in both cases). In case of Tau, lithium enhanced its plasma concentration in an insignificant way $(\mathrm{p}=0.1744)$. Similar to the case of Met, selenium, given both alone and in co-treatment with lithium, did not change the plasma concentration ( $\mathrm{p}=0.7343$ and 0.9997 , respectively) (Fig. 2A). Plasma concentrations of Tau and Met showed a positive correlation with Spearman correlation factor $\mathrm{R}=0.6484 ; \mathrm{p}=0.0006$ (Fig. 4A).

Plasma Phe was enhanced by lithium in a significant way $(p=0.0060)$ when compared to control. Selenium, both given alone and in co-treatment with lithium, changed plasma concentration, but the observed increase was not significant ( $p=0.1904$ and 0.3989 , respectively). In the case of Tyr, the observed increase vs. control in animals given lithium was not significant $(\mathrm{p}=0.1016)$.The selenium-administered groups also showed an increase vs. control, but this effect was not so considerable (Se alone $-\mathrm{p}=1.0000$; Se in co-treatment with lithium $-\mathrm{p}=0.6681$ ) (Fig. 2B). Plasma concentrations of Phe and Tyr showed a positive correlation with Spearman correlation factor $\mathrm{R}=0.8785$ and $\mathrm{p}=0.0000$ (Fig. 4B).

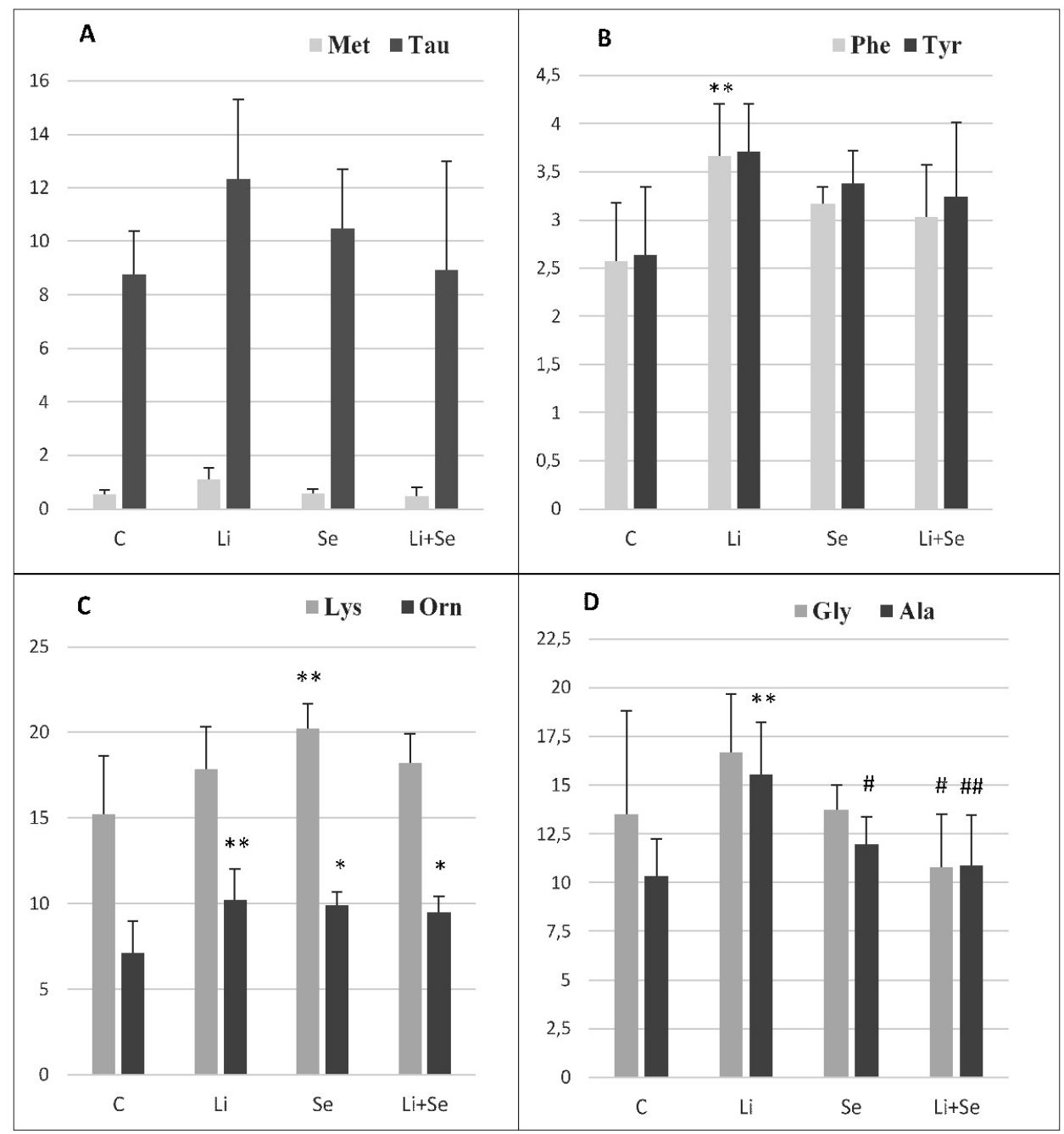

Figure 2. Plasma concentrations $\left[\mathrm{mmol} / \mathrm{dm}^{3}\right]$ of chosen amino acids in rats treated with lithium, selenium or both elements together. Values are mean $\pm S D$. ${ }^{*} p<0.05$ vs. control; ${ }^{* *} p<0.01$ vs. control; $\# p<0.05$ vs. Li-group; \#\# $<0.01$ vs. Li-group 


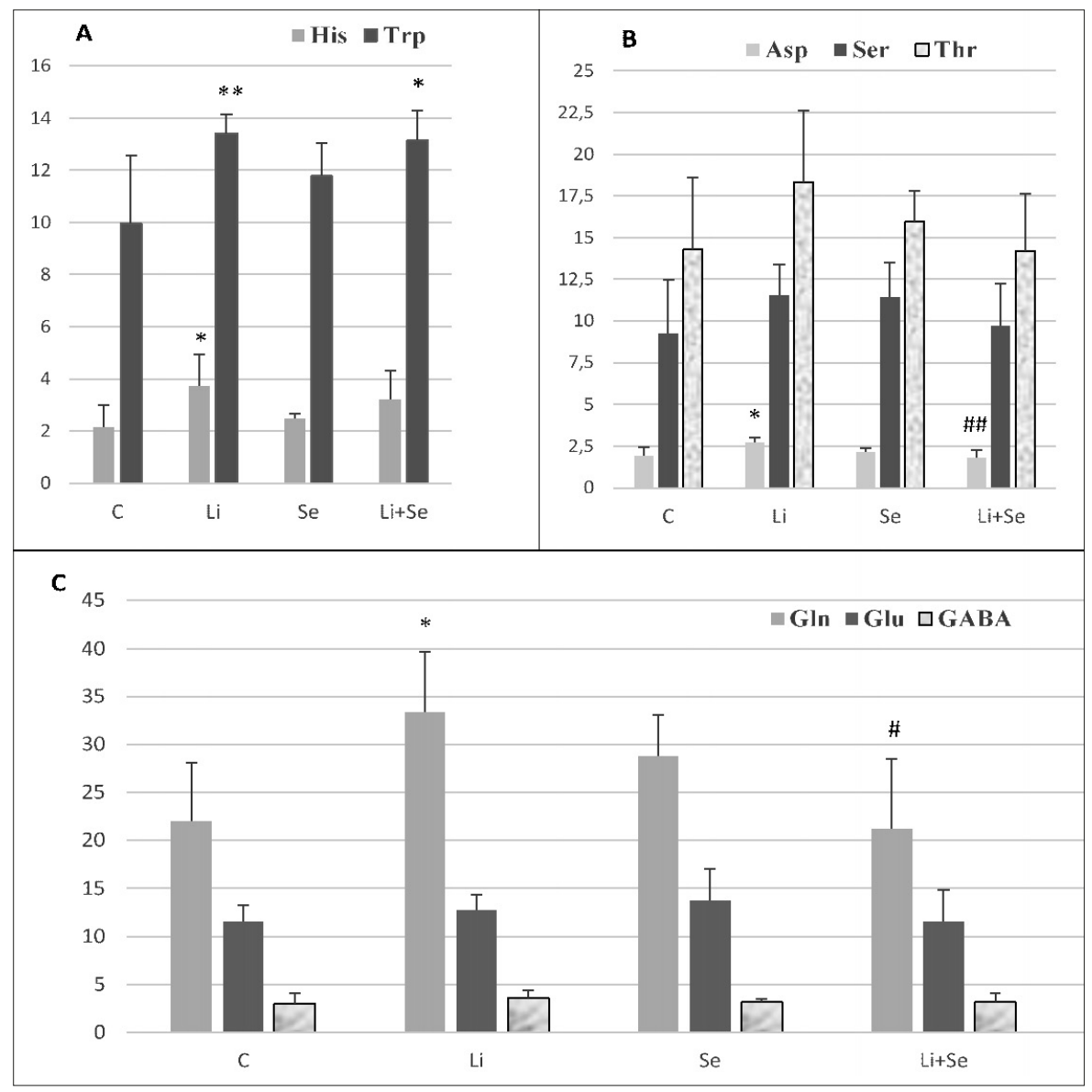

Figure 3. Plasma concentrations $\left[\mathrm{mmol} / \mathrm{dm}^{3}\right]$ of chosen amino acids in rats treated with lithium, selenium or both elements together. Values are mean \pm SD. ${ }^{*} \mathrm{p}<0.05$ vs. control; ${ }^{* *} \mathrm{p}<0.01$ vs. control; $\# \mathrm{p}<0.05$ vs. Li-group; $\# \#$ p 0.01 vs. Li-group

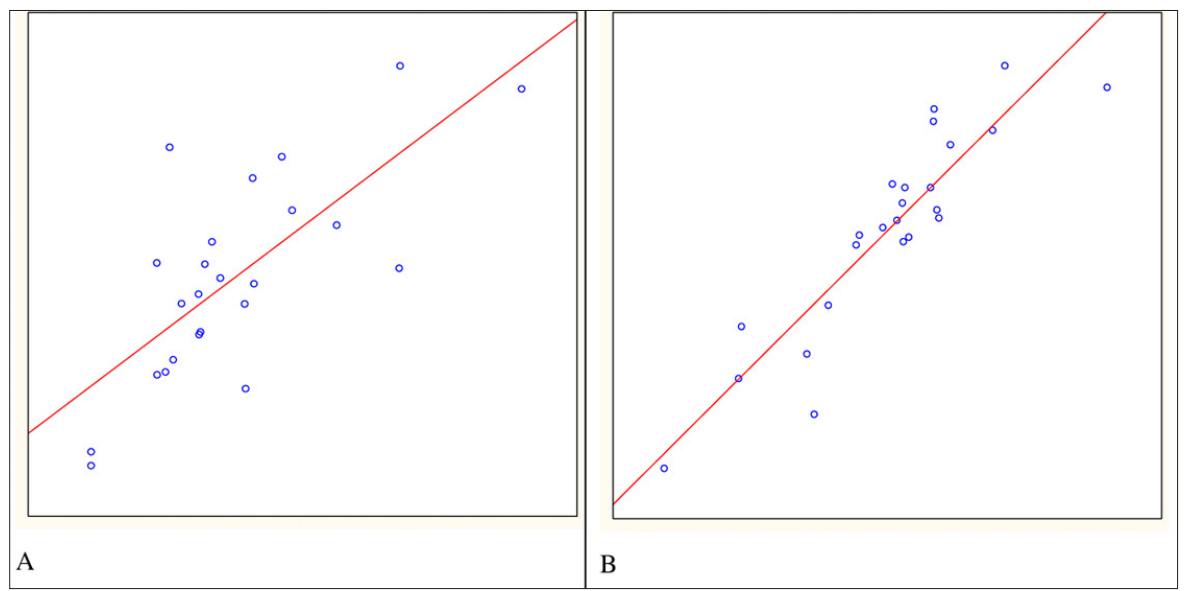

Figure 4. Correlations between Tau and Met (A) and between Phe-Tyr (B)

Lys plasma concentration was not affected markedly by $\mathrm{Li}$ alone ( $\mathrm{p}=0.7855$ vs. control) and $\mathrm{Li}+$ Se treatment $(\mathrm{p}=0.6148$ vs. control). Surprisingly, Se alone caused a significant increase compared to control animals $(\mathrm{p}=0.0076)$. Orn was enhanced in comparison to control in all studied groups (Li alone $-\mathrm{p}=0.0069$; Se alone $-\mathrm{p}=0.0156 ; \mathrm{Li}+\mathrm{Se}$-group $\mathrm{p}=0.0473$ ) (Fig. 2C).

Gly concentration in plasma was insignificantly enhanced by Li treatment vs. control $(\mathrm{p}=0.3969)$. The values obtained for $\mathrm{Li}$ alone and $\mathrm{Li}+\mathrm{Se}$ groups differed significantly $(\mathrm{p}=0.0326)$. Se administration did not cause any changes vs. control $(\mathrm{p}=0.9994)$, while in the $\mathrm{Li}+\mathrm{Se}$ group a slight decrease compared to control animals was observed $(\mathrm{p}=0.5154)$. Similarly, Ala concentration in plasma was increased by lithium vs. control, but in this case in a significant way $(\mathrm{p}=0.0031)$. Statistically significant differences were also found between $\mathrm{Li}$ alone and the Se alone groups $(\mathrm{p}=0.0482)$, as well as between $\mathrm{Li}$ alone and $\mathrm{Li}+\mathrm{Se}$ groups ( $\mathrm{p}=0.0078)$. Se-administration, alone and together with lithium, caused no well-marked changes compared to control $(\mathrm{p}=0.5959$ and $\mathrm{p}=0.9756$, respectively) (Fig. 2D).

Plasma His concentration was increased vs. control in all studied groups, but this effect reached significance only in 
the case of Li-treated rats $(\mathrm{p}=0.0320)$. In the Se-group and $\mathrm{Li}+\mathrm{Se}$-group this effect was not well marked $(\mathrm{p}=0.9012$ and $\mathrm{p}=0.2213$ vs. control, respectively). Trp was also enhanced vs. control, but in this case lithium, given both alone and in cotreatment with selenium, caused a well-marked effect, while Se alone only an insignificant effect $(\mathrm{p}=0.0062$ for Li alone, 0.2267 for Se alone, and $\mathrm{p}=0.0120$ for $\mathrm{Li}+\mathrm{Se}$-rats) (Fig. 3A).

Plasma Asp concentration was significantly increased by the administration of $\mathrm{Li}$ alone ( $\mathrm{p}=0.0118 \mathrm{vs}$. control). In the groups given selenium, both alone and together with $\mathrm{Li}$, the differences vs. control did not reach significance $(p=0.7274$ and $\mathrm{p}=0.9674$, respectively). The values obtained in $\mathrm{Li}$ alone and $\mathrm{Li}+\mathrm{Se}$ treated groups differed significantly $(\mathrm{p}=0.0043)$. In the case of Ser, no statistically significant differences were observed. However, in animals treated with $\mathrm{Li}$ and Se alone, the average concentrations were higher than in control ( $\mathrm{p}=0.4056$ and 0.4409 , respectively). In contrast, in the $\mathrm{Li}+\mathrm{Se}$ administered group, the noted value did not differ from control $(p=0.9899)$. Thr plasma concentration in the studied groups did not differ markedly compared to the control group ( $p$ values $0.2422,0.8555$ and 0.9999 for $\mathrm{Li}, \mathrm{Se}$ and $\mathrm{Li}+\mathrm{Se}$ groups, respectively) (Fig. 3B).

Gln in plasma was markedly enhanced in the Li alone group ( $\mathrm{p}=0.0115 \mathrm{vs}$. control) and insignificantly in Se alone $(\mathrm{p}=0.2474$ vs. control). No difference was found between control and $\mathrm{Li}+\mathrm{Se}$-treated rats $(\mathrm{p}=1.0000)$. There was a significant difference between $\mathrm{Li}$ and $\mathrm{Li}+\mathrm{Se}$ treated groups $(\mathrm{p}=0.0151)$. Plasma Glu was insignificantly enhanced vs. control in Li and Se alone given animals ( $p=0.8763 ; \mathrm{p}=0.5040$ ). Again, no differences were found between control and $\mathrm{Li}+\mathrm{Se}$ treated rats $(p=1.0000)$. GABA in turn was insignificantly increased in the Li alone group ( $p=0.6604$ vs. control), and practically unchanged in Se and $\mathrm{Li}+\mathrm{Se}$ animals $(\mathrm{p}=0.9949$; 0.9950, respectively) (Fig. 3C).

\section{DISCUSSION}

The current study concerned the changes in selected amino acids concentration in the plasma of rats receiving selenium and/or lithium. Selection of the amino acids was dictated mainly by their physiological role, as well by their relationships with the studied elements. Many of them act as neurotransmitters themselves or make their precursors. GABA, produced from glutamate, glycine and taurine, belong to inhibitory neurotransmitters while glutamate and aspartate belong to excitatory neurotransmitters [30]. Another important neurotransmitter, serotonin, is synthesized from tryptophan [31]. Phenylalanine in turn, converted into tyrosine, produces the precursor of catecholamines [32]. Histidine is a precursor of histamine, a biogenic amine which plays numerous physiological roles [33]. Serine has been found to be connected with selenium metabolism [34]. The connections between enzymes involved in amino acids (alanine and aspartate) metabolism and lithium exposure has also been stated [35].

In the presented study, selenium administration caused an increase in the plasma level of the selected amino acids, although this effect reached statistical significance only in the case of lysine and ornithine. The lack of a distinct connection between selenium treatment and different amino acids profile is essentially consistent with observations reported by other researchers in both human and animal studies.
The lack of relationships between selenium and total homocysteine concentration in plasma was found by Venn et al., who reported that in human subjects with suboptimal Se status who received selenium supplementation for 20 weeks, an increase in selenium was not accompanied by any change in total homocysteine [36]. Nevertheless, contradicting results have also been reported. González et al. observed that in elderly subjects from nursing homes, serum selenium was associated with total homocysteine in an inverse way [37]. In another study, the influence of the potential lithium-mimetic organic selenocompound ebselen (3600 mg over 24 hours), administered to healthy volunteers, on selected metabolites in the anterior cingulate cortex and occipital cortex was studied. The results differed, depending on the region studied. In the anterior cingulate cortex, Glu, Gln and Glx (a composite of glutamate and glutamine) were decreased compared to a placebo group, while GABA was unaltered. In turn, in the occipital cortex, none of the mentioned parameters were affected [16].

$\mathrm{Qu}$ et al. investigated the possible protective effect of sodium selenite against cadmium chloride toxicity, with both elements administered in the diet. Cadmium significantly decreased the content of the selected amino acids (valine, leucine, arginine and proline) in chicken pectoral muscle, and selenium showed a distinct restoring effect. However, selenium itself did not show any significant influence on the content of most of the studied amino acids [12].

Research on the effect of dietary selenium in the form of sodium selenite, given in combination with either vitamin E or vitamin E + oleuropein (a phenolic compound of antioxidant properties occurring in olive oils) to pigs for 35 days, revealed that among 19 studied plasma amino acids, only cystine and lysine were affected; the former being enhanced and the latter decreased [38].

No direct connection was found between glutamine and selenium levels in the blood in humans suffering from different diseases $[39,40]$. Animal studies also revealed no relationships between serum selenium and plasma amino acids profile in pathological states [41].

The results presented above show that selenium generally has little effect on the level of amino acids in the organism, in both the physiological and pathological states. This could be connected to the fact that enzymes involved into amino acid metabolism do not seem to be much influenced by selenium treatment. Elemental selenium in the form of nanoparticles (SeNPs) given to rats was found to cause a decrease in serum ALT while AST remained unchanged [42]. Ansar et al. did not observe any effect of sodium selenite - the form used in the current study - on both these enzymes in rats serum [43].

However, in the light of the latest research, the issue of the mutual relationships between Se and amino acids becomes more complicated, because dietary serine supplementation to sows resulted in no change of serum selenium, while serum selenoprotein P was increased [34]. The small effect of selenium treatment on amino acids plasma profile seems to be of importance considering its application in agriculture, as well as the still growing interest in its supplementation both in pathological and physiological states.

In the current study, lithium treatment generally increased the concentrations of all the studied amino acids. In the available databases there are few reports on the relationships between amino acids profile in blood and lithium treatment. The comparison is all the more difficult because the reported 
animal studies often greatly differed from the current study in the method of administration, treatment time, and doses.

Pettegrew et al. studied the influence of intraperitoneal administration of lithium chloride on phospholipids and amino acids in brain of rats. The authors found that intraperitoneal Li-treatment for 14 days $(2 \mathrm{mmol} / \mathrm{kg}$ ) decreased brain taurine by $8 \%$ and increased aspartate by $9 \%$. Additionally, a very slight enhancement of glutamine and alanine was observed, while glutamate remained practically unchanged [44]. McFarlane et al. examined the tyrosine level in the serum and striatum of male rats during three hours following acute intraperitoneal treatment $(3 \mathrm{mEq} / \mathrm{kg})$ with lithium chloride. In serum, tyrosine was decreased in a significant way after one and 1.5 hours, while the striatal level was found to be depressed after 1.5 and two hours [20]. Bhalla et al. observed no effect of dietary lithium carbonate given for two months on cerebrum and cerebellum L-citrulline in rats [45].

The results of the performed human studies also showed some discrepancies. As they included patients with bipolar disorder, dependence was observed of Li influence on the responsiveness of the patients. Friedmann et al. reported that in bipolar disorder subjects receiving lithium, grey matter Glx (glutamate + glutamine + GABA) was decreased, an effect that was positively correlated with the Li level in serum [46]. Zanetti et al. studied the effect of lithium therapy in subjects suffering from bipolar disorder and receiving Li therapy for six weeks on selected metabolites in the left hippocampus. Although no significant difference in glutamate level before and after treatment was found, statistical analysis revealed very interesting relationships between the lithium concentration in plasma after six weeks and changes of hippocampal glutamate over time. Lower $\mathrm{Li}(0.2-0.49 \mathrm{mmol} / \mathrm{L})$ was connected with a reduction in Glu level, and higher Li $(\geq 0.5 \mathrm{mmol} / \mathrm{L})$ with an overall enhancement of Glu [47].

The levels of amino acids in the plasma of bipolar disorder patients subjected to lithium therapy was also studied, while additionally taking lithium responsiveness into account. Compared to the healthy control, patients showed a decrease in different amino acids, including Val, Leu, Tyr, Phe, Glu, Pro and Lys, while Trp remained unchanged. Phe and Tyr were decreased in Li non-responders vs. healthy control, while in Li responders no alteration of those amino acids compared to control was observed. Interestingly, no significant differences between non-responders and responders regarding Phe and Tyr were noted, while Pro was lower in the responders group. However, it must be considered that non-responders were also administered other mood stabilizers [22].

The alterations of amino acids caused by lithium administration could also be connected with Li effect on aminotransferases. In the current study, both Ala and Asp were increased in Li-treated animals. Ben Saad et al. observed an enhancement of ALT and AST in the serum of rats receiving lithium carbonate [35]. However, in this case, contradicting outcomes were also reported by Park et al. who did not observe any changes of these enzymes in obese rats given $\mathrm{LiCl}$ [48].

The results concerning the effect of lithium administration on amino acids plasma profile could contribute to the research regarding the mechanism of Li action, which seems to be important considering the growing concern about the possibility of environmental exposure to this element.
In the presented study, co-treatment with $\mathrm{Li}+\mathrm{Se}$ generally resulted in a slight or no influence, except for a well-marked enhancement in the case of tryptophan and ornithine. It seems to be worthwhile to investigate further why only the mentioned amino acids were influenced to the greatest extend. These facts are all the more interesting considering that tryptophan is a precursor of serotonin, and lithium has been found to affect the serotonin pathway. Moreover, ornithine takes part in the urea cycle, and kidney disturbances are known to be connected with lithium treatment [49].

\section{CONCLUSIONS}

The current study has shown the significant effect of lithium alone on plasma amino acids profile in experimental animals, and the much less influence of selenium alone while cotreatment generally resulted in a slight or no effect at all. The need for further research aimed at resolving the problem of mutual relationships and influence of lithium and selenium on amino acids profile, seems to be warranted all the more because, to the best of the authors' knowledge, the reports on the influence of co-treatment with these two elements are scarce. Moreover, the slight influence of selenium seems to be of importance regarding its agricultural application and the still growing interest in its supplementation, while the results concerning lithium could contribute to research regarding the mechanism of Li action.

\section{REFERENCES}

1. Trippe RC 3rd, Pilon-Smits EAH. Selenium transport and metabolism in plants: Phytoremediation and biofortification implications. J Hazard Mater. 2021; 404(Pt B): 124178. https://doi.org/10.1016/j. jhazmat.2020.124178

2. Kieliszek M. Selenium-Fascinating Microelement, Properties and Sources in Food. Molecules. 2019; 24(7): 1298. doi: 10.3390/ molecules 24071298

3. Andrade FR, da Silva GN, Guimarães KC, et al. Selenium protects rice plants from water deficit stress. Ecotoxicol Environ Saf. 2018; 164: 562-570. doi: 10.1016/j.ecoenv.2018.08.022. https://doi.org/10.1016/j. ecoenv.2018.08.022

4. Jóźwiak W, Politycka B. Effect of Selenium on Alleviating Oxidative Stress Caused by a Water Deficit in Cucumber Roots. Plants (Basel). 2019; 8(7): 217. doi: 10.3390/plants8070217. https://doi.org/10.3390/ plants8070217

5. Rayman MP. Selenium intake, status, and health: a complex relationship. Hormones (Athens). 2020; 19(1): 9-14. doi: 10.1007/s42000-019-00125-5

6. Gangadoo S, Dinev I, Willson NL, et al. Nanoparticles of selenium as high bioavailable and non-toxic supplement alternatives for broiler chickens. Environ Sci Pollut Res Int. 2020; 27(14): 16159-16166. doi: 10.1007/s11356-020-07962-7

7. Liang N, Wang F, Peng X, et al. Effect of Sodium Selenite on Pathological Changes and Renal Functions in Broilers Fed a Diet Containing Aflatoxin B . Int J Environ Res Public Health. 2015; 12(9): 11196-11208. https://doi.org/10.3390/ijerph120911196

8. Ramkissoon C, Degryse F, da Silva RC, et al. Improving the efficacy of selenium fertilizers for wheat biofortification. Sci Rep. 2019; 9(1): 19520. doi: 10.1038/s41598-019-55914-0. Erratum in: Sci Rep. 2020; 10(1): 16963. https://doi.org/10.1038/s41598-019-55914-0

9. Mehta SQ, Behl S, Day PL, et al. Evaluation of $\mathrm{Zn}, \mathrm{Cu}$, and Se Levels in the North American Autism Spectrum Disorder Population. Front Mol Neurosci. 2021; 14: 665686. https://doi.org/10.3389/fnmol.2021.665686

10. Scassellati C, Bonvicini C, Benussi L, et al. Neurodevelopmental disorders: Metallomics studies for the identification of potential biomarkers associated to diagnosis and treatment. J Trace Elem Med Biol. 2020; 60: 126499. https://doi.org/10.1016/j.jtemb.2020.126499

11. Constantinescu-Aruxandei D, Frîncu RM, Capră L, et al. Selenium Analysis and Speciation in Dietary Supplements Based on Next- 
Generation Selenium Ingredients. Nutrients. 2018; 10(10): 1466. doi: $10.3390 /$ nu10101466

12. Qu KC, Li HQ, Tang KK, et al. Selenium Mitigates Cadmium-Induced Adverse Effects on Trace Elements and Amino Acids Profiles in Chicken Pectoral Muscles. Biol Trace Elem Res. 2020; 193(1): 234-240. https:// doi.org/10.1007/s12011-019-01682-x

13. Rasinger JD, Lundebye AK, Penglase SJ, et al. Methylmercury Induced Neurotoxicity and the Influence of Selenium in the Brains of Adult Zebrafish (Danio rerio). Int J Mol Sci. 2017; 18(4): 725. https://doi. org/10.3390/ijms18040725

14. Solovyev ND. Importance of selenium and selenoprotein for brain function: From antioxidant protection to neuronal signalling. J Inorg Biochem. 2015; 153: 1-12. https://doi.org/10.1016/j.jinorgbio.2015.09.003

15. Antoniadou I, Kouskou M, Arsiwala T, et al. Ebselen has lithium-like effects on central 5-HT2A receptor function. Br J Pharmacol. 2018; 175(13): 2599-2610. https://doi.org/10.1111/bph.14179

16. Masaki C, Sharpley AL, Godlewska BR, et al. Effects of the potential lithium-mimetic, ebselen, on brain neurochemistry: a magnetic resonance spectroscopy study at 7 tesla. Psychopharmacology (Berl). 2016; 233(6): 1097-1104. doi: 10.1007/s00213-015-4189-2

17. Masaki C, Sharpley AL, Cooper CM, et al. Effects of the potential lithium-mimetic, ebselen, on impulsivity and emotional processing. Psychopharmacology (Berl). 2016; 233(14): 2655-2661. doi: 10.1007/ s00213-016-4319-5

18. Boga Pekmezekmek A, Emre M, Tunc E, et al. L-Glutamic acid monosodium salt reduces the harmful effect of lithium on the development of Xenopus laevis embryos. Environ Sci Pollut Res Int. 2020; 27(33): 42124-42132. https://doi.org/10.1007/s11356-020-10155-X

19. Rybakowski JK. Lithium treatment in the era of personalized medicine. Drug Dev Res. 2020; https://doi.org/10.1002/ddr.21660

20. McFarlane HG, Steele J, Vinion K, et al. Acute lithium administration selectively lowers tyrosine levels in serum and brain. Brain Res. 2011; 1420: 29-36. doi: 10.1016/j.brainres.2011.08.054

21. Farooq M, Kim S, Patel S, et al. Lithium increases synaptic GluA2 in hippocampal neurons by elevating the $\delta$-catenin protein. Neuropharmacology. 2017; 113(Pt A): 426-433. https://doi.org/10.1016/j. neuropharm.2016.10.025

22. Joshi MB, Pai S, Balakrishnan A, et al. Evidence for perturbed metabolic patterns in bipolar disorder subjects associated with lithium responsiveness. Psychiatry Res. 2019; 273: 252-259. https:// doi.org/10.1016/j.psychres.2019.01.031

23. Wakita M, Nagami H, Takase Y, et al. Modifications of excitatory and inhibitory transmission in rat hippocampal pyramidal neurons by acute lithium treatment. Brain Res Bull. 2015; 117: 39-44. doi: 10.1016/j. brainresbull.2015.07.009

24. Kubo H, Nakataki M, Sumitani S, et al. 1H-magnetic resonance spectroscopy study of glutamate-related abnormality in bipolar disorder. J Affect Disord. 2017; 208: 139-144. https://doi.org/10.1016/j. jad.2016.08.046

25. Sarawagi A, Soni ND, Patel AB. Glutamate and GABA Homeostasis and Neurometabolism in Major Depressive Disorder. Front Psychiatry. 2021;12: 637863. https://doi.org/10.3389/fpsyt.2021.637863

26. Teo CH, Soga T, Parhar I. Lithium chloride enhances serotonin induced calcium activity in EGFP-GnIH neurons. Sci Rep. 2020; 10(1): 13876. https://doi.org/10.1038/s41598-020-70710-x

27. An Y, Inoue T, Kitaichi Y, et al. Combined treatment with subchronic lithium and acute intracerebral mirtazapine microinjection into the median raphe nucleus exerted an anxiolytic-like effect synergistically. Eur J Pharmacol. 2016; 783: 112-116. https://doi.org/10.1016/j. ejphar.2016.05.004

28. Harari F, Bottai M, Casimiro E, et al. Exposure to Lithium and Cesium Through Drinking Water and Thyroid Function During Pregnancy: A Prospective Cohort Study. Thyroid. 2015; 25(11): 1199-1208. doi: 10.1089/thy.2015.0280

29. Meshram P, Mishra A, Abhilash, et al. Environmental impact of spent lithium ion batteries and green recycling perspectives by organic acids - A review. Chemosphere. 2020; 242: 125291. doi: 10.1016/j. chemosphere.2019.125291

30. Leenaars CHC, Drinkenburg WHP, Nolten C, et al. Sleep and Microdialysis: An Experiment and a Systematic Review of Histamine and Several Amino Acids. J Circadian Rhythms. 2019; 17: 7. https:// doi.org/10.5334/jcr.183

31. Shah PA, Park CJ, Shaughnessy MP, et al. Serotonin as a Mitogen in the Gastrointestinal Tract: Revisiting a Familiar Molecule in a New Role. Cell Mol Gastroenterol Hepatol. 2021: S2352-345X(21)00097-7. https://doi.org/10.1016/j.jcmgh.2021.05.008

32. Dinu A, Apetrei C. A Review on Electrochemical Sensors and Biosensors Used in Phenylalanine Electroanalysis. Sensors (Basel). 2020; 20(9): 2496. doi: 10.3390/s20092496

33. Moriguchi T, Takai J. Histamine and histidine decarboxylase: Immunomodulatory functions and regulatory mechanisms. Genes Cells. 2020; 25(7): 443-449. https://doi.org/10.1111/gtc.12774

34. Zhou L, Feng Y, Liu Y, et al. Serine Supplementation in the Diets of Late Gestating and Lactating Sows Improves Selenium Nutritional Status in Sows and Their Offspring. Biol Trace Elem Res. 2021; https://doi. org/10.1007/s12011-021-02661-x

35. Ben Saad A, Dalel B, Rjeibi I, et al. Phytochemical, antioxidant and protective effect of cactus cladodes extract against lithium-induced liver injury in rats. Pharm Biol. 2017; 55(1): 516-525. https://doi.org/1 $0.1080 / 13880209.2016 .1255976$

36. Venn BJ, Grant AM, Thomson CD, et al. Selenium supplements do not increase plasma total homocysteine concentrations in men and women. J Nutr. 2003; 133(2): 418-420. https://doi.org/10.1093/jn/133.2.418

37. González S, Huerta JM, Alvarez-Uría J, et al. Serum selenium is associated with plasma homocysteine concentrations in elderly humans. J Nutr. 2004; 134(7): 1736-1740. https://doi.org/10.1093/jn/134.7.1736

38. Rey AI, de-Cara A, Calvo L, et al. Changes in Plasma Fatty Acids, Free Amino Acids, Antioxidant Defense, and Physiological Stress by Oleuropein Supplementation in Pigs Prior to Slaughter. Antioxidants (Basel). 2020; 9(1): 56. https://doi.org/10.3390/antiox9010056

39. Yang CJ, Huang TS, Lee TL, et al. Serum Glutamine Levels as a Potential Diagnostic Biomarker in Sepsis following Surgery for Peritonitis. Chin J Physiol. 2017; 60(6): 320-326. doi: 10.4077/CJP.2017.BAG495

40. Jang JY, Lee SH, Shim H, et al. Serum oxygen radical activity and total antioxidation capacity are related with severities of surgical patient with sepsis: Prospective pilot study. J Crit Care. 2017; 39: 131-136. https:// doi.org/10.1016/j.jcrc.2017.01.016

41. Sabatino BR, Rohrbach BW, Armstrong PJ, et al. Amino acid, iodine, selenium, and coat color status among hyperthyroid, Siamese, and age-matched control cats. J Vet Intern Med. 2013; 27(5): 1049-1055. https://doi.org/10.1111/jvim.12165

42. Al-Quraishy S, Dkhil MA, Abdel Moneim AE. Anti-hyperglycemic activity of selenium nanoparticles in streptozotocin-induced diabetic rats. Int J Nanomedicine. 2015; 10: 6741-6756. https://doi.org/10.2147/ IJN.S91377

43. Ansar S, Alshehri SM, Abudawood M, et al. Antioxidant and hepatoprotective role of selenium against silver nanoparticles. Int J Nanomedicine. 2017; 12: 7789-7797. https://doi.org/10.2147/IJN. S136748. Erratum in: Int J Nanomedicine. 2018; 13: 5769.

44. Pettegrew JW, Panchalingam K, McClure RJ, et al. Effects of chronic lithium administration on rat brain phosphatidylinositol cycle constituents, membrane phospholipids and amino acids. Bipolar Disord. 2001; 3(4): 189-201. https://doi.org/10.1034/j.1399-5618.2001.30404.x

45. Bhalla P, Singla N, Dhawan DK. Potential of lithium to reduce aluminium-induced cytotoxic effects in rat brain. Biometals. 2010; 23(2): 197-206. doi: 10.1007/s10534-009-9278-4

46. Friedman SD, Dager SR, Parow A, et al. Lithium and valproic acid treatment effects on brain chemistry in bipolar disorder. Biol Psychiatry. 2004; 56(5): 340-348. https://doi.org/10.1016/j.biopsych.2004.06.012

47. Zanetti MV, Otaduy MC, de Sousa RT, et al. Bimodal effect of lithium plasma levels on hippocampal glutamate concentrations in bipolar II depression: a pilot study. Int J Neuropsychopharmacol. 2014; 18(6): pyu058. doi: 10.1093/ijnp/pyu058

48. Park J, Cheon W, Kim K. Effects of Long-Term Endurance Exercise and Lithium Treatment on Neuroprotective Factors in Hippocampus of Obese Rats. Int J Environ Res Public Health. 2020; 17(9): 3317. https:// doi.org/10.3390/ijerph17093317

49. Davis J, Desmond M, Berk M. Lithium and nephrotoxicity: Unravelling the complex pathophysiological threads of the lightest metal. Nephrology (Carlton). 2018; 23(10): 897-903. https://doi.org/10.1111/ nep. 13263 